\title{
Thermally Stimulated Depolarization Current from HCl-Doped Ice
}

\author{
Yoon Young Choi \\ Group of Phoenix, Kangwon Science High School, San 1, Taejang 1 dong, Wonju, Kangwondo 220-121, Republic of Korea \\ Correspondence should be addressed to Yoon Young Choi, yychoi1215@naver.com
}

Received 15 March 2011; Revised 19 April 2011; Accepted 20 June 2011

Academic Editor: Lorenzo Pavesi

Copyright () 2011 Yoon Young Choi. This is an open access article distributed under the Creative Commons Attribution License, which permits unrestricted use, distribution, and reproduction in any medium, provided the original work is properly cited.

Thermally stimulated depolarization current (TSDC) technique has been applied to investigate the dielectric properties of the $\mathrm{HCl}$-doped ice. Three TSDC peaks were found from HCl-doped ice as well as ice of pure water. Among three peaks, the $\alpha$ and $\gamma$ peaks are affected strongly by the concentrations of $\mathrm{HCl}$. We found that the $\mathrm{pH}$ numbers are linearly proportioned to the ratios of the areas of the peaks. The relation between $x_{\alpha}$ (the ratio of the area of $\alpha$ peak to the area of $\beta$ peak) and $\mathrm{pH}_{\mathrm{HCl}, \alpha} \mathrm{was}_{\mathrm{found}}$ to be $\mathrm{pH}_{\mathrm{HCl}, \alpha}=7.77-2.86 x_{\alpha}$. This result suggests the possibility of the development of an apparatus to measure the $\mathrm{pH}$ number of acid contaminated water. The activation energies correspond to depolarization processes reflected in the $\alpha, \beta$, and $\gamma$ peaks that were found to be $267 \pm 19 \mathrm{meV}, 431 \pm 30 \mathrm{meV}$, and $635 \pm 44 \mathrm{meV}$, respectively.

\section{Introduction}

Water is one of the most abundant substances in nature and essential to human's life and industries. Polluted water will cause a serious threat of life in nature and industries. Despite stricter grovel environmental regulations, frequency of acid rain increases as industry grows, and impacts of acid rain in forest degradation, surface water contamination, and corrosion of building materials become a serious environmental problem. Therefore it is important to investigate dielectric property of acid polluted water [1].

If a strong electric field is applied to a dielectric material, dipoles in the material will be arranged in a certain direction and the materialis polarized. If that material is cooled down while applying the electric field, the arranged dipoles are frozen in a metastable state and stay in ordered phase even if the electric field is removed. If the material is warmed up, the material is depolarized by thermal energy. During the depolarization process, current flows in the external circuit. This current is called the thermally stimulated depolarization current (TSDC) [2]. In case of a perfect crystal phase dielectric material it is hard to rearrange dipoles by an external electric field. It is known that in crystals with vacancies, interstitials or impurity ions of other types dipolar complexes are formed near impurities. Such dipolar defects are the origin of the TSDC. This TSDC method has been extensively used for many crystals that contain dipolar defects that can be oriented by an external electric field [3-8].

In this paper we studied dielectric properties of $\mathrm{HCl}$ polluted ice using TSDC technique. We found that there are three peaks in TSDS measurement as a function of temperature which indicate existence of three metastable sites of dipoles in ice. The area of the peaks is logarithmically proportional to concentration of $\mathrm{HCl}$.

The reported TSDC results show that it is very sensitive to the materials contained in water $[9,10]$. Therefore, TSDC technique is also good for investigating the physical properties such as the trap density of water dipole in the electric field, the activation energy of the trapped water dipole, and the relation between TSDC peak area and the concentration of the $\mathrm{HCl}$ in the water. This technique can be applied to the development of the dosimeter for the various physical or chemical quantities. But this technique has no practical use because there are relative simple methods for 
similar measurements, and the TSDC method is relatively expensive and complex. Further study may enable this technique to be practical.

\section{Experiment}

$\mathrm{HCl}$-doped water samples were prepared by diluting $10 \%$ $\mathrm{HCl}$ with deionized water to the target concentrations. To measure TSDC, a Pt parallel-plate capacitor was used in this experiment. The dimension of the parallel-plate was $10 \mathrm{~mm} \times 10 \mathrm{~mm}$, and the distance between two plates was $1 \mathrm{~mm}$. The experimental apparatus (the capacitor and a liquid nitrogen tank) was shielded by an aluminum box to reduce electrical noise. This shielding also helps in keeping steady heating rate for each experiment. After filling the capacitor with the liquid sample at room temperature, the capacitor was cooled down to $220 \mathrm{~K}$, and electric field was applied to the capacitor for 1 minute. Then the capacitor was immersed in liquid nitrogen to cool the sample down to liquid nitrogen temperature while the electric field was applied. The polling voltage is $1000 \mathrm{~V}$ which is equivalent to 1.0 MV/m electric field applied to the sample. During the polling treatment the current in the circuit was less than $0.1 \mathrm{nA}$.This means that the liquid sample was not electrically decomposed. After reaching the liquid nitrogen temperature, the two ends of the lead wire were connected to the $\mathrm{Pt}$ plates to discharge residue charge induced during the polling treatment. After discharging, the capacitor was taken out of liquid nitrogen tank, and TSDC was measured as a function of temperature using a current amplifier and a voltage amplifier connected to the A-D converter. The heating rate of the sample was $\eta=0.9759-0.0028 T$ in $\mathrm{K} / \mathrm{sec}$ units where $T$ is the temperature of the sample in Kelvin degree.

\section{Results and Analysis}

It is known that ice has Ih structure at 1 atm and temperature $80 \mathrm{~K}-273 \mathrm{~K}$, and ice of Ih structure has $\mathrm{H}-\mathrm{O}$ bonding axes [11]. Water molecules in pure ice are tightly bound to each other to form crystal, and it is hard to polarize the molecules by the external electric field. However if there are broken bonds due to defects or impurities water molecules can be oriented along the external electric field. By cooling to much lower temperature this metastable ordered phase may be locked in. This is the origin of the TSDC peaks in the water ice.

Figure 1 shows TSDC graphs as a function of temperature for ice doped by the various concentrations of $\mathrm{HCl}$. In this figure, the currents are normalized to compare the relative intensities of the peaks. In the case of pure water as well as $\mathrm{HCl}$-doped water, three peaks appeared at $110 \mathrm{~K}$, $130 \mathrm{~K}$, and $160 \mathrm{~K}$. The presence of three peaks means that there are three kinds of defects in the ice made of pure or $\mathrm{HCl}$ - doped water, or there are excited states for a dipole in the electric field. The TSDC from pure water shows that the $\beta$ peak was strong and the $\alpha$ and $\gamma$ peaks were relatively weak. Note the $\beta$ peak of $1.0 \times 10^{-3} \%$ mole concentration is smaller than that of $2.5 \times 10^{-5} \%$ mole concentration. This is because of the volume of the sample solutions. However,

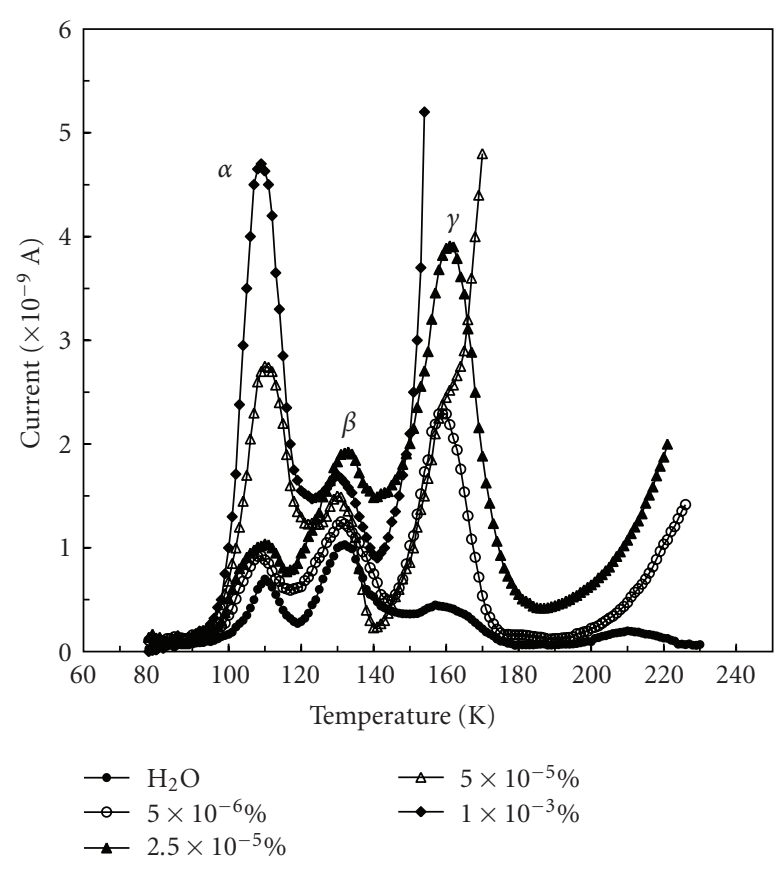

FIGURE 1: TSDC graphs from the ice doped with various concentrations of $\mathrm{HCl}$. As increasing the concentration of $\mathrm{HCl}$, the $\alpha$ and $\gamma$ peaks become stronger and the $\beta$ peak is affected weekly by the concentration of $\mathrm{HCl}$. The strong currents for the concentrations $5.0 \times 10^{-6} \%$ and $2.5 \times 10^{-5} \%$ at the temperature higher than $200 \mathrm{~K}$ are due to the space charges. For the concentration $1.0 \times 10^{-3} \%$, the $\gamma$ peaks are overlapped by the space charge current.

with an increasing concentration of $\mathrm{HCl}$, the $\alpha$ and $\gamma$ peaks became stronger, and no peak appeared except these three peaks.

On the other hand, the $\mathrm{H}^{+}$and the $\mathrm{Cl}^{-}$ions can be strongly hydrated, with water dipoles arranged spherically around the ions. This hydration would form a new type of crystal, and it would make a new TSDC peak. However, as shown in this figure, a new TSDC peak was not found in the $\mathrm{HCl}$-doped ice. Therefore it is concluded that the hydrations induced by the $\mathrm{H}^{+}$or the $\mathrm{Cl}^{-}$ions do not make new type defect. As described above, the $\beta$ peak of the ice made of pure water was the strongest. However, as the concentration of $\mathrm{HCl}$ increased, the $\alpha$ and $\gamma$ peaks became stronger than the $\beta$ peak. This occurrence of this phenomenon means that the dipoles inducing the $\beta$ peak were more stable than those inducing the $\alpha$ and $\gamma$ peaks. So to speak, the $\mathrm{H}^{+}$or $\mathrm{Cl}^{-}$ions affect strongly on the densities of defects (broken $\mathrm{H}-\mathrm{O}$ bondings) inducing the $\alpha$ and $\gamma$ peaks but affect relatively weekly on the density of defect inducing the $\beta$ peak.

Various methods were tried to investigate the relationship between the $\mathrm{pH}$ number and TSDC peaks. Because the TSDC depends on the volume of the sample solution and the electrodes surrounding the sample solution, it is difficult to determine the relationship between the TSDC and the $\mathrm{pH}$ number. Figure 1 shows that the areas of peaks were strongly affected on the concentration of $\mathrm{HCl}$. Therefore, the areas of peaks were focused to find the relationship. 
In the $\mathrm{HCl}$-doped water, some portion of the $\mathrm{H}^{+}$or $\mathrm{Cl}^{-}$ ions would contribute to make the defects, and some portion of $\mathrm{H}^{+}$or $\mathrm{Cl}^{-}$ions would remain as the space charge. If we set $n$ as the number of defectsin the iceper unit volume, $C$ as the concentration of $\mathrm{HCl}, \Delta C$ as the infinitesimal concentration of $\mathrm{HCl}$ contributes to make the infinitesimal defect $\Delta n$ then, $\Delta C$ is proportion alto $C$ and $\Delta n$, that is, $\Delta C=A C \Delta n$. Here, $A$ is a proportional constant. This equation can be expressed as following integral form. The negative sign means that increment of $\Delta C$ results in decrement of $C$,

$$
\int_{C_{0}}^{C} \frac{1}{C} d C=-\int_{n_{0}}^{n} d n
$$

The result of (1) is

$$
\ln C=\ln C_{0}-A n_{0}-A n .
$$

The unit of $\ln C$ is the unit of $\mathrm{pH}$ number. This equation reveals that $\mathrm{pH}$ concentration is linearly proportional to the number of the defects. The equation (2) can be written as follows:

$$
\mathrm{pH}=B-A n .
$$

Here, $B$ is a constant.

To investigate the relationship between the concentration of $\mathrm{HCl}$ and TSDC peaks, the areas of peaks were estimated using curve fitting method. It was found that the number of the defects is not proportional to the intensity of the TSDC peak, but it is proportional to the area of the TSDC peak. With try and error, we found the consistent relationship. As explained above, the $\beta$ peak is relatively stable and affected weekly by $\mathrm{HCl}$. Therefore the area of the $\beta$ peak was chosen as a reference area. It is found that the ratios of the areas of the $\alpha$ peaks to the areas of the $\beta$ peaks were linearly proportional to the $\mathrm{pH}$ concentration. Figure 2 shows the relationship between the $\mathrm{pH}$ concentrations of $\mathrm{HCl}$ and the ratios of the areas of the $\alpha$ peaks to the areas of the $\beta$ peaks.

If we let $x_{\alpha}$ be the ratio of areas (the area of the $\alpha$ peak divided by the area of the $\beta$ peak), the $\mathrm{pH}$ concentration estimated by the $\alpha$ peak is given by following equation:

$$
\mathrm{pH}_{\mathrm{HCl}, \alpha}=7.77-2.86 x_{\alpha} \text {. }
$$

This result is in good agreement with the theoretical equation shown in (3). It is expected that the similar analysis for the $\gamma$ peaks would show similar result as (1). But as shown in Figure 1, the $\gamma$ peak at the high concentration of $\mathrm{HCl}$ was too strong to estimate the area. However, because of the lack of the data points, the similar analysis was not tried for the $\gamma$ peaks. However, if we measure the ratios of the areas of peaks, we can determine the $\mathrm{pH}$ numbers without considering the volume of samples. Therefore this result suggests the possibility of the development of the $\mathrm{pH}$ dosimeter using TSDC technique.

Although it is known that there are at least three hydrogen bonds per water molecule [11], the number of TSDC peaks present depended on the experimental conditions. The structure of interfacial water differs from that of bulk water

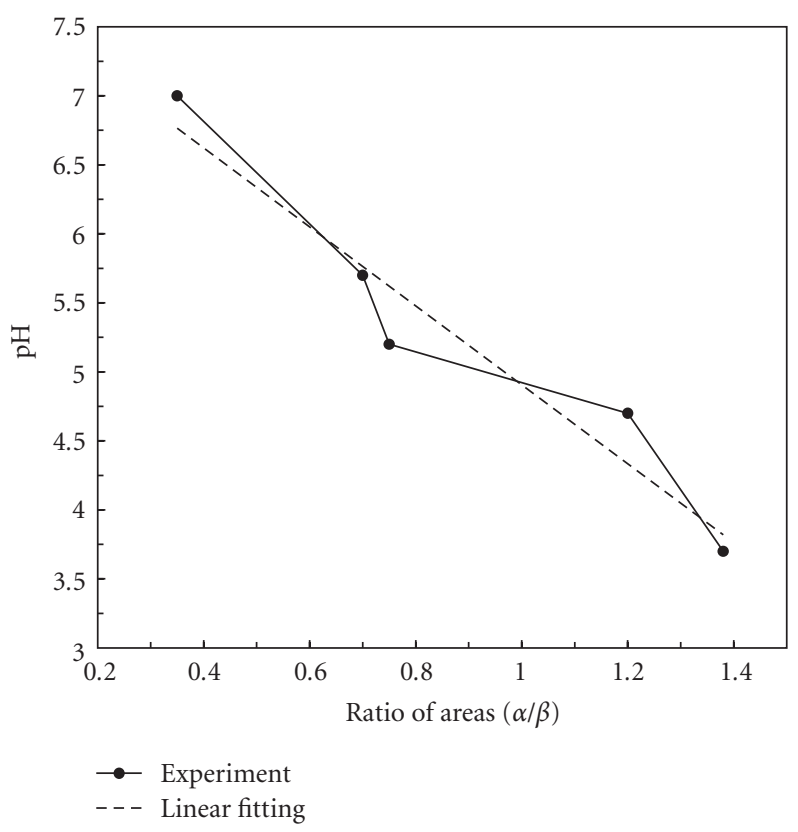

FIgURE 2: The relationship between the $\mathrm{pH}$ concentrations of $\mathrm{HCl}-$ doped water and the ratio of areas (the area of the $\alpha$ peak divided by the area of the $\beta$ peak). Although the data points are slightly scattered, the ratios of the peak areas are linearly proportional to the $\mathrm{pH}$ concentrations of $\mathrm{HCl}$. The dashed line is the linear fit.

because of changes in the hydrogen bond network at a phase boundary, depending on the nature of the second phase. The number of TSDC peaks and the intensities of the individual peaks should be dependent on the electrode surrounding the water. Gun'ko and his coworker found only two TDSC peaks from water. They used water samples absorbed in a porous $\mathrm{SiO}_{2}$ absorbent tablet. In this study the water samples were placed in a Pt parallel-plate capacitor. There should be more defects on the surface of the water than in the bulk of the water. The physical properties of the surface are quite different from those of the bulk, and the physical properties of a water surface in contact with $\mathrm{SiO}_{2}$ should also be different from those of a water surface in contact with Pt. Therefore, the type of defects present at the interface between water and $\mathrm{SiO}_{2}$ may be different from those at the interface between water and Pt. The different interfaces may shift the peaks, the peak intensities, or the number of peaks. Gun'ko also indicated that the interaction of water with solid surfaces results in the displacement of peaks. Riehl [11] found three strong peaks at $120 \mathrm{~K}, 130 \mathrm{~K}$, and $171 \mathrm{~K}$ and a weak peak at $140 \mathrm{~K}$. Although they did not indicate the type of material used for the electrode surrounding the ice made of pure water, their results were similar to these results. The difference between Bishop's results and these results may have come from the different heating rate and the different materials used for the electrode. In this case, the water molecules were in contact with Pt plates. Therefore, it is to be expected that these TSDC results from water were different from other results. 


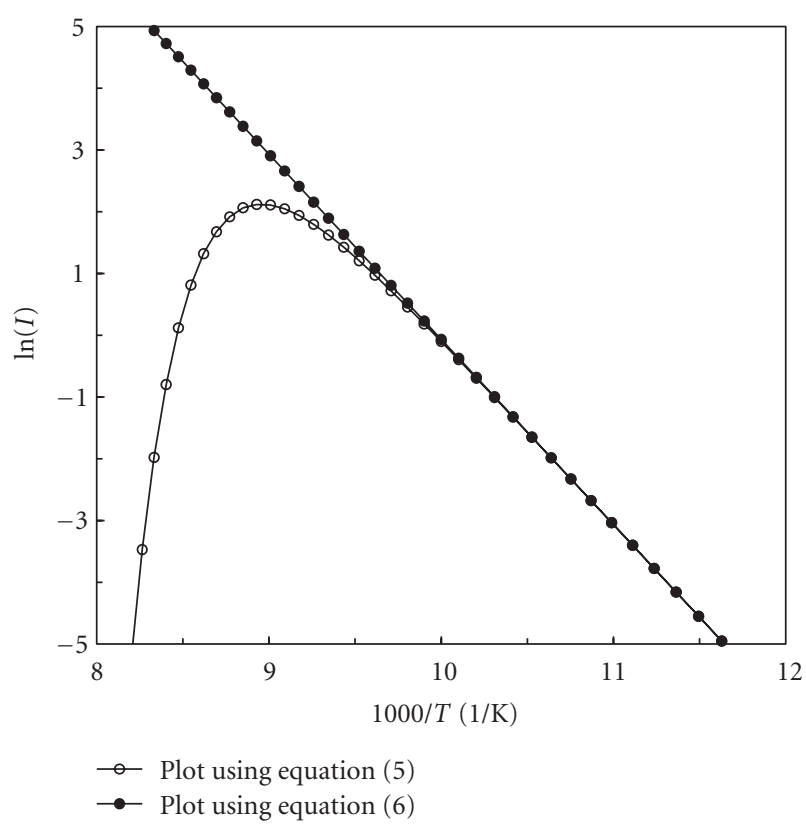

Figure 3: Arrhenius plots against 1000/T. Open circles denote the graph plotted using (5), and small closed circles denote the graph plotted using (6). The slopes of two graphs in the temperature region $85 \mathrm{~K} \sim 100 \mathrm{~K}$ are almost same.

It is well known that the thermally stimulated current is given by a general equation [12],

$$
I(T)=A \exp \left[-\frac{E_{t}}{k T}-\frac{B}{\eta} \int_{T_{0}}^{T} \exp \left(-\frac{E_{t}}{k T}\right) d T\right]
$$

where parameters $A$ and $B$ are constant, $E_{t}$ is the activation energy, $\eta$ is the heating rate, $T_{0}$ is the initial temperature, and $T$ will be $T_{0}+\eta t$. If $T_{0} \approx T$, (5) can be written as

$$
\ln (I)=\ln (A)-\frac{E_{t}}{k T} .
$$

The slope of the plot of $\ln (I)$ versus $1 / T$ reveals the activation energy. This approximation can accompany some error. To check the error range due to this approximation, two slopes obtained using two theoretical curves plotted using (5) and (6) are compared. Figure 3 shows two semilogarithm graphs plotted using (5) and the graph plotted using (6).These plots correspond to the $\alpha$ peak in Figure 1.

In the temperature region $85 \mathrm{~K} \sim 100 \mathrm{~K}$, this figure reveals that the slope of the graph plotted using (5) is bigger by $0.2 \%$ than that of the graph plotted using (6). But the experimental error was found to be $\sim 5 \%$. Therefore the error occurred by the approximation can be ignored, and so (6) is a good approximation in the low temperature region of a peak. It is expected that the activation energy would change according to the concentration of $\mathrm{HCl}$. However, the activation energy does not change. This means that the interaction between $\mathrm{H}^{+}$or $\mathrm{Cl}^{-}$ion and water molecules is not strong enough to change the activation energy of the water dipole. The activation energies correspond to depolarization processes reflected in the $\alpha, \beta$, and $\gamma$ peaks in Figure 1 calculated using (6) which are $267 \pm 19 \mathrm{meV}, 431 \pm 30 \mathrm{meV}$, and $635 \pm 44 \mathrm{meV}$. Bishop and his coworkers reported that the activation energy of the peak appearing at $120 \mathrm{~K}$ is $250 \pm 20 \mathrm{meV}$. This value is in good agreement with this result. Reports describing the activation energies of other peaks were not found. However, we think that these results are reasonable.

\section{Conclusion}

In summary, the dielectric properties of the $\mathrm{HCl}$-doped ice have been investigated using TSDC technique. Three TSDC peaks were found that for the $\mathrm{HCl}$-doped ice as well as the pure water ice. It is found $\mathrm{H}^{+}$or $\mathrm{Cl}^{-}$ion does not make new type defect (broken hydrogen bond) in the ice. The $\alpha$ peak and the $\gamma$ peak are affected strongly, but the $\beta$ peak is affected weakly by the concentrations of $\mathrm{HCl}$. The relation between $\mathrm{x}_{\alpha}$ (the ratio of the area of the $\alpha$ peak to the area of the $\beta$ peak) and the $\mathrm{pH}$ concentration of $\mathrm{HCl}$-doped water was found to be $\mathrm{pH}_{\mathrm{HCl}, \alpha}=7.77-2.86 X_{\alpha}$. The activation energies correspond to depolarization processes reflected in the $\alpha, \beta$ and $\gamma$ peaks which are found to be $267 \pm 19 \mathrm{meV}$, $431 \pm 30 \mathrm{meV}$ and $635 \pm 44 \mathrm{meV}$.

\section{Acknowledgments}

This work was supported by the Korea Foundation for the Advancement of Science and Creativity (KOFAC) through the $R \& E$ project.

\section{References}

[1] H. J. C. Blume, "Measurement of dielectric properties and determination of microwave emissivity of polluted waters," IEEE Transactions on Instrumentation and Measurement, vol. IM-29, no. 4, pp. 289-291, 1980.

[2] R. Chen and Y. Kirsh, Analysis of Thermally Stimulated Process, Pergamon Press, 1981.

[3] I. Guler and N. M. Gasanly, "Determination of trapping center parameters in $\mathrm{Tl}_{2} \mathrm{In}_{2} \mathrm{~S}_{3}$ Se layered single crystals by thermally stimulated current measurements," Physica B, vol. 404, no. 1415, pp. 2034-2038, 2009.

[4] V. M. Gun'ko, V. V. Turov, V. I. Zarko, E. V. Goncharuk, and A. A. Turova, "Regularities in the behaviour of water confined in adsorbents and bioobjects studied by $1 \mathrm{H}$ NMR spectroscopy and TSDC methods at low temperatures," Colloids and Surfaces A, vol. 336, no. 1-3, pp. 147-158, 2009.

[5] S. U. Haq, S. H. Jayaram, E. A. Cherney, and G. G. Raju, "Space charge analysis in enamelled wires by using thermally stimulated depolarization current (TSDC)," Journal of Electrostatics, vol. 67, no. 1, pp. 12-17, 2009.

[6] P. Tewari, R. Rajagopalan, E. Furman, and M. T. Lanagan, "Control of interfaces on electrical properties of $\mathrm{SiO} 2-$ Parylene-C laminar composite dielectrics," Journal of Colloid and Interface Science, vol. 332, no. 1, pp. 65-73, 2009.

[7] N. Souffi, G. H. Bauer, C. Main, S. Reynolds, and R. Brüggemann, "Density of states in the gap of microcrystalline silicon determined from thermally-stimulated currents," Thin Solid Films, vol. 516, no. 20, pp. 6844-6847, 2008.

[8] T. Yildirim and N. M. Gasanly, "Thermally stimulated current measurements in as-grown TlGaSeS layered single crystals," Current Applied Physics, vol. 9, no. 6, pp. 1278-1282, 2009. 
[9] V. M. Gun’ko, V. I. Zarko, E. V. Goncharuk et al., "TSDC spectroscopy of relaxational and interfacial phenomena," Advances in Colloid and Interface Science, vol. 131, no. 1-2, pp. 1-89, 2007.

[10] V. A. Tischenko and V. M. Gun'ko, "Water electret relaxation at dispersed silica surfaces," Colloids and Surfaces A, vol. 101, no. 2-3, pp. 287-294, 1995.

[11] N. Riehl, Physics of Ice, Pelnum, New York, NY, USA, 1969.

[12] S. Yadav, R. K. Pal, S. K. Sharma, and A. Kumar, "Determination of trap depth and trap density in $\mathrm{Se}_{70} \mathrm{Te}_{30-x} \mathrm{Zn}_{x}$ thin films using thermally stimulated current measurements," Physica B, vol. 404, no. 16, pp. 2225-2228, 2009. 

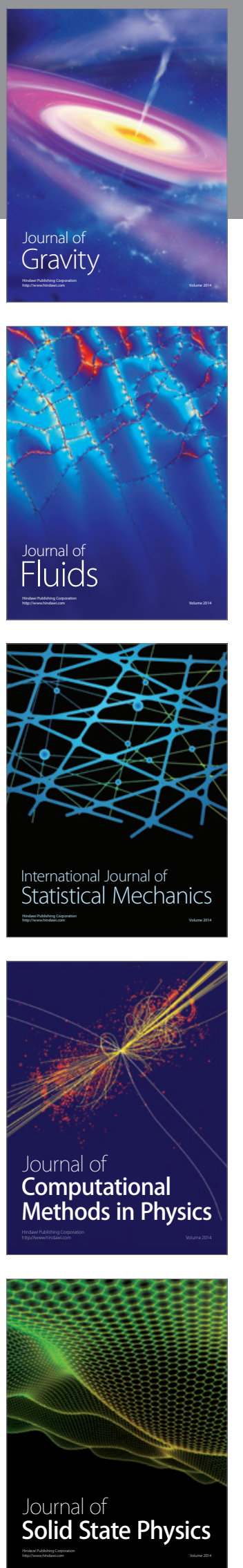

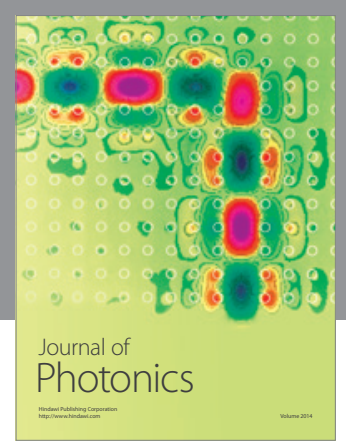

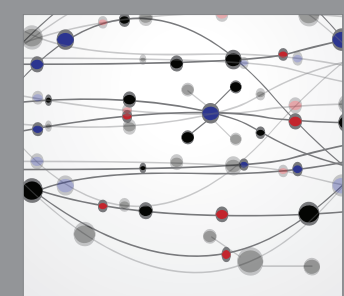

The Scientific World Journal
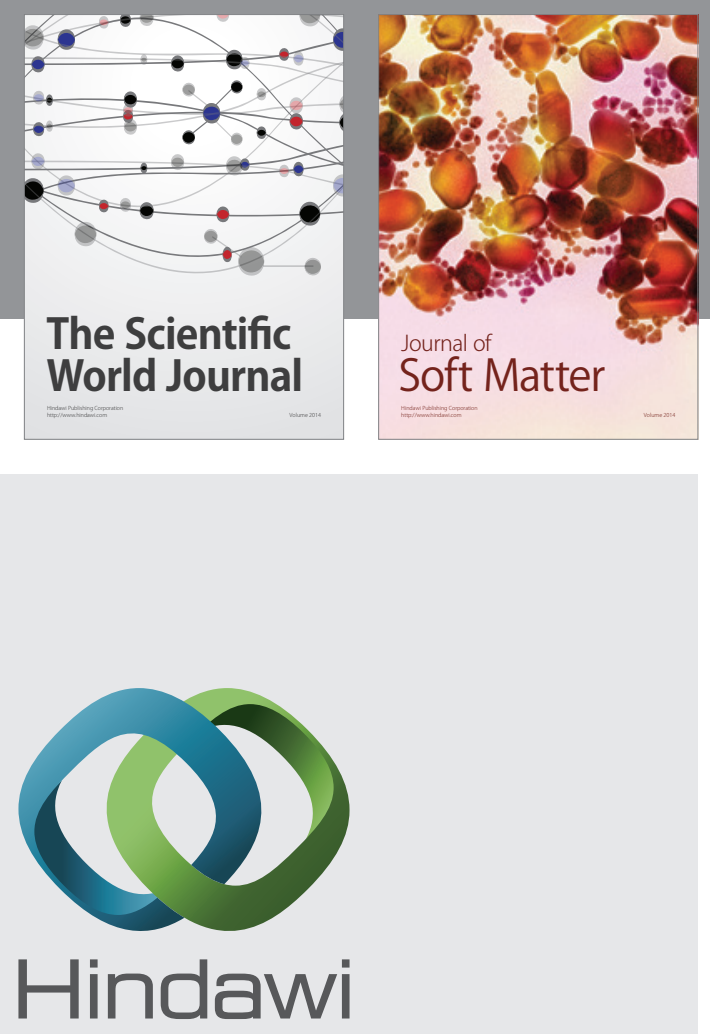

Submit your manuscripts at

http://www.hindawi.com
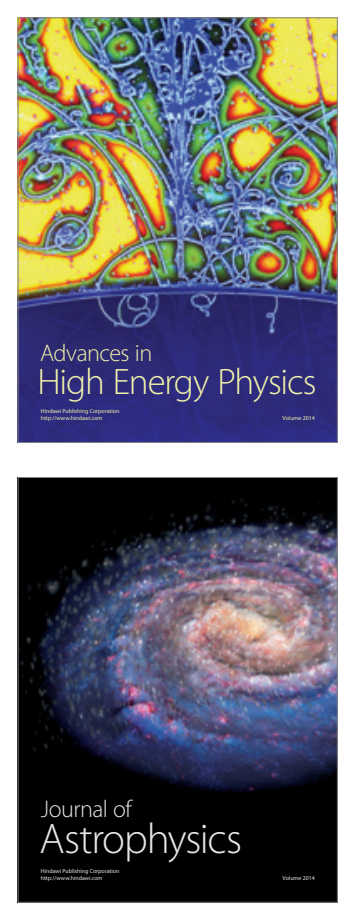
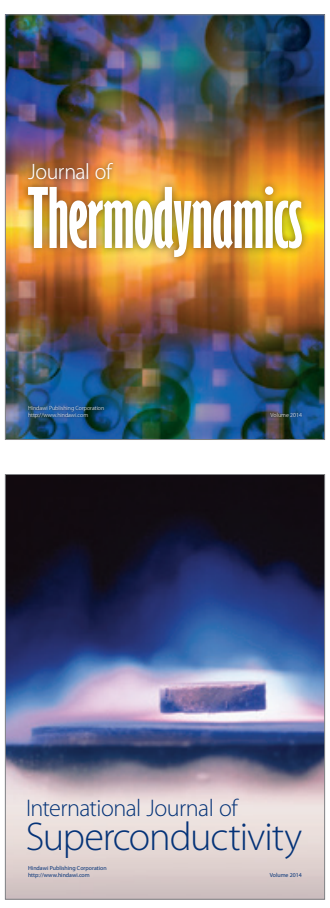
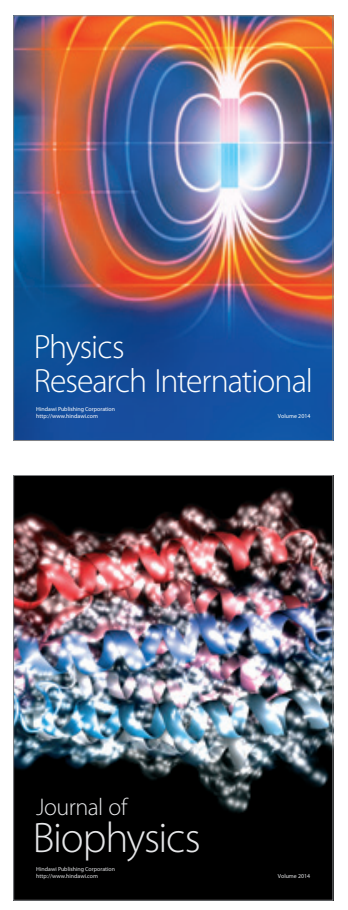
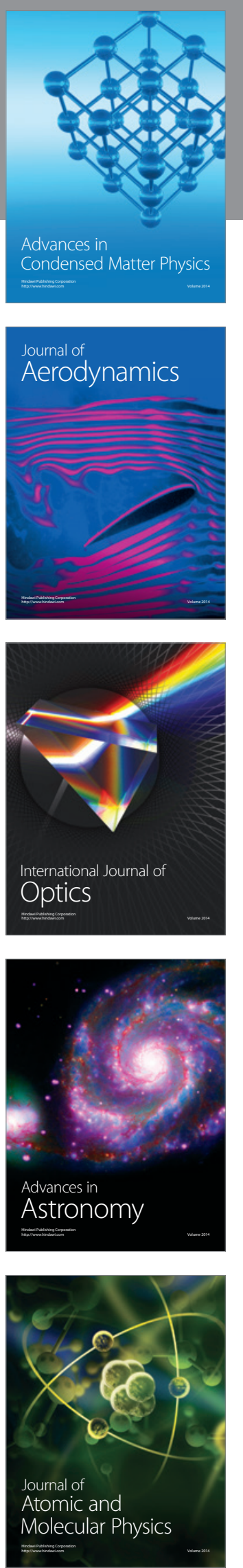